Review Article

\title{
Mitochondrial Dysfunction in Intervertebral Disc Degeneration: From Pathogenesis to Therapeutic Target
}

\author{
Danni Li, ${ }^{1}$ Fenghua Tao, ${ }^{2}$ and Lin Jin $\mathbb{D}^{2}$ \\ ${ }^{1}$ Department of Anesthesiology, Renmin Hospital of Wuhan University, Wuhan 430060, China \\ ${ }^{2}$ Department of Orthopedics, Renmin Hospital of Wuhan University, Wuhan 430060, China \\ Correspondence should be addressed to Lin Jin; jinlin2010@whu.edu.cn
}

Received 5 September 2020; Revised 16 November 2020; Accepted 17 November 2020; Published 27 November 2020

Academic Editor: Sidong Yang

Copyright ( 2020 Danni Li et al. This is an open access article distributed under the Creative Commons Attribution License, which permits unrestricted use, distribution, and reproduction in any medium, provided the original work is properly cited.

\begin{abstract}
Mitochondria are cytosolic organelles essential for cellular function and survival. The function of mitochondria is maintained by mitochondrial quality control systems including mitochondrial fission and fusion to adapt the altered environment and mitophagy for removal of damaged mitochondria. Mitochondrial dysfunction is closely involved in aging-related diseases. Intervertebral disc (IVD) degeneration, an aging-associated process, is the major contributor to low back pain. Growing evidence has suggested that the mitochondrial function in IVD cells is severely compromised during the degenerative process of IVD, and dysfunctional mitochondria along with impaired mitochondrial dynamics and mitophagy cause a series of cascade reactions that have been implicated in increased oxidative stress, senescence, matrix catabolism, and apoptosis of IVD cells, thereby contributing to the degeneration of IVD. Accordingly, therapies that target mitochondrial dysfunction and related mechanisms, such as ROS generation, mitophagy, and specific molecules and signaling, hold great promise. The present review summarizes the current state of the role of mitochondrial dysfunction in the pathophysiology of IVD degeneration and potential therapeutic strategies that could be developed.
\end{abstract}

\section{Introduction}

The intervertebral disc (IVD) is a fibrocartilaginous tissue interspacing and connecting adjacent vertebrae, which serves to absorb and transmit mechanical loading from the spine and permits movement of the spine [1]. The IVD consists of three components. The central gelatinous nucleus pulposus (NP) primarily contains NP cells, type II collagen, and proteoglycan. The outer annulus fibrosus (AF) is mainly composed of AF cells and type I collagen fibers. The cartilaginous endplate (CEP) is a hyaline cartilaginous tissue that joins IVD with the adjacent bony vertebrae [2]. NP cells act a critical role in producing extracellular matrix (ECM) components including type II collagen and proteoglycan, maintaining the integrity and homeostasis of IVD [3].

Low back pain (LBP) is globally prevalent and more than $80 \%$ of people will suffer from LBP during their lifetime [4, 5]. It is also the main cause of disability, causing heavy socioeconomic cost each year worldwide $[4,6]$. Degeneration of the IVD characterized by advanced signs of aging and pro- gressive structural destruction is widely recognized as a major contributor to LBP [7]. Although factors including obesity, genetic, trauma, and lifestyle (e.g., sedentary work and the lack of sports activities) are associated with the development of IVD degeneration $[8,9]$, the exact cellular and molecular mechanisms underlying IVD degeneration are complex and multifactorial. From past decades, extensive evidence has suggested that oxidative stress, apoptosis, and metabolic dysregulation of disc-resident cells, especially in NP cells, are closely involved in the pathogenesis of IVD degeneration [10]. Therefore, some intracellular regulatory approaches that control these cellular processes are extremely important and receive considerable attention.

Mitochondria are the double membrane, cytoplasmic organelles with their own genome, the mitochondrial DNA (mtDNA). Mitochondria play a crucial role in energy production, mainly through the mechanism of oxidative phosphorylation [2]. In addition, these organelles contribute to key biochemical processes including the generation of reactive oxygen species (ROS) [11] and the regulation of calcium 
homeostasis [12], which affect cell metabolism, function, and survival. The mitochondrial quality control system, including mitochondrial fission and fusion, and selective self-clearance mechanism termed mitophagy, enables them to modify their shape, size, quality, and quantity in response to the cellular needs and various stresses, thereby maintaining mitochondrial and cellular homeostasis [13, 14]. Given its essential roles in cellular processes, it is not surprising that dysfunction or impairment of mitochondria will be implicated in a series of pathological processes, such as oxidative stress, senescence, apoptosis, and ECM degradation, consequently causing detrimental effects on cell function and survival. As we know, a wide range of diseases covering metabolic disorders, cancer, and inflammatory diseases, particularly in degenerative diseases, is characterized by the involvement of mitochondrial dysfunction [15-18].

In recent years, the role of mitochondria in IVD degeneration is widely studied, and these studies have yielded a more comprehensive understanding of the pathophysiology of IVD degeneration and provided some promising therapeutic approaches for the treatment of IVD degeneration, which we aim to summarize and discuss in this review.

\section{Mitochondrial Function and Homeostasis}

As energy factories in mammal cells, mitochondria are responsible for producing energy. Additionally, these organelles are vital regulators of redox state and calcium balance. A quality control machinery of mitochondria, referring to mitochondrial dynamics and mitophagy, serves for the maintenance of mitochondrial homeostasis to fulfill their function. These processes proceed properly under the physiological conditions, whereas aberrant stresses or stimulus will cause mitochondrial dysfunction, subsequently leading to a series of cascade reactions (Figure 1).

2.1. Energy Metabolism, Redox, and Calcium Regulation. In mammal cells, mitochondria are the sites of the tricarboxylic acid (TCA) cycle and oxidative phosphorylation (OXPHOS). These "factories" produce energy using the electrochemical gradient generated across the inner of two membranes by the electron transport chain (ETC) [19]. There exists a special mitochondrial membrane system to perform productive work of energy. Inner mitochondrial membrane (IMM) surrounds the mitochondrial matrix and takes in the electrons produced by the TCA cycle through ETC. The ETC, located at IMM, contains a number of proteins, for example, complex I-IV, that perform the transfer and incremental release of energy from the electrons in order to pump protons $\left(\mathrm{H}^{+}\right)$ into the intermembrane space and establish the electrochemical gradient. This mitochondrial membrane potential $(\Delta \psi \mathrm{m})$ drives the process of OXPHOS, providing energy by converting ADP to ATP [20]. Although residing in a low oxygen tension environment, IVD cells are not entirely anaerobic and still carry out oxidative metabolism [21, 22].

Mitochondria continuously metabolize oxygen and meantime generate ROS. During the transportation of electrons, a small part of electrons will leak and reduce oxygen to superoxide anion $\left(\mathrm{O}_{2}{ }^{-}\right)$, termed "primary" $\mathrm{ROS}$ [23]. The
$\mathrm{O}_{2}{ }^{-}$could be further converted to the "secondary" ROS including hydrogen peroxide $\left(\mathrm{H}_{2} \mathrm{O}_{2}\right)$ and hydroxyl radical $(\mathrm{OH}-)$ via enzymatic and nonenzymatic mechanisms. The mitochondrial-dependent ROS (mtROS) generation has been found in multiple disc-resident cells including NP cells of humans and rats and AF cells of humans and rats [24-27]. A proper level of ROS is fundamental to organismal physiology and adaptive responses [28], which relies on a balance between ROS generation and ROS scavenge by nonenzymatic and enzymatic antioxidants. The interruption of this balance will induce oxidative stress that is detrimental to the mitochondrial function and cells [29].

Mitochondria also play a vital role in regulating cellular calcium $\left(\mathrm{Ca}^{2+}\right)$ homeostasis. The cytosolic $\mathrm{Ca}^{2+}$ is taken into mitochondria by energy-driven uniporters, while the efflux of $\mathrm{Ca}^{2+}$ from the mitochondria is mediated by ion gradientdriven antiporters [30]. The mitochondrial permeability transition pore (mPTP) is a high-conductance, voltage- and $\mathrm{Ca}^{2+}$-sensitive channel, whose opening can be transient to physiologically allow quick release of $\mathrm{Ca}^{2+}$ and/or metabolite exchange between the mitochondrial matrix and the cytosol [31]. However, mitochondrial $\mathrm{Ca}^{2+}$ overload, especially when combined with oxidative stress and/or ATP depletion, will trigger a drastic efflux of $\mathrm{Ca}^{2+}$ through the prolonged opening of $\mathrm{mPTP}$, consequently causing dissipation of the $\Delta \psi \mathrm{m}$, respiratory chain uncoupling, cessation of ATP synthesis, ROS generation, and eventually mitochondrial swelling, rupture, and cell death [31].

2.2. Mitochondrial Dynamics and Mitophagy. Mitochondria are dynamic organelles, continually undergoing fission and fusion for regulating their number, size, and distribution [32]. Mitochondrial fission is a multistep process allowing the division of one mitochondrion in two mitochondria that are controlled by proteins such as dynamin-related protein 1 (DRP1), mitochondrial fission factor (Mff), and fission 1 (Fis1) [33]. Mitochondrial fusion is the combination of two mitochondria with the outer mitochondrial membrane (OMM) fusion mediated by mitofusins (MFN1 and MFN2) followed by IMM fusion, determined by optic atrophy 1 (OPA1) [34]. These balanced dynamic transitions are required not only to ensure mitochondrial function but also to respond to meet cellular metabolic demands and adapt the nutrient condition [13]. In addition, mitochondrial dynamics is engaged in repairing damaged mitochondria [35]. If these processes fail to restore mitochondria in a healthy state, the damaged mitochondria will be eliminated by a selective autophagy mechanism termed mitophagy.

Mitophagy is involved in the basal mitochondrial turnover and quality control via the clearance of long-lived or damaged mitochondria [36]. Different stimulus and conditions induce mitophagy in distinct mechanisms. Generally, mitophagy can be divided into Parkin RBR E3 ubiquitinprotein ligase- (Parkin-) dependent and Parkinindependent pathways. When mitochondria are depolarized under various cellular stress, the PINK1 accumulates on OMM and subsequently recruits and activates Parkin [37]. The Parkin polyubiquitylates multiple OMM proteins, which will be recognized by LC3 adaptors, such as P62 and NDP52 


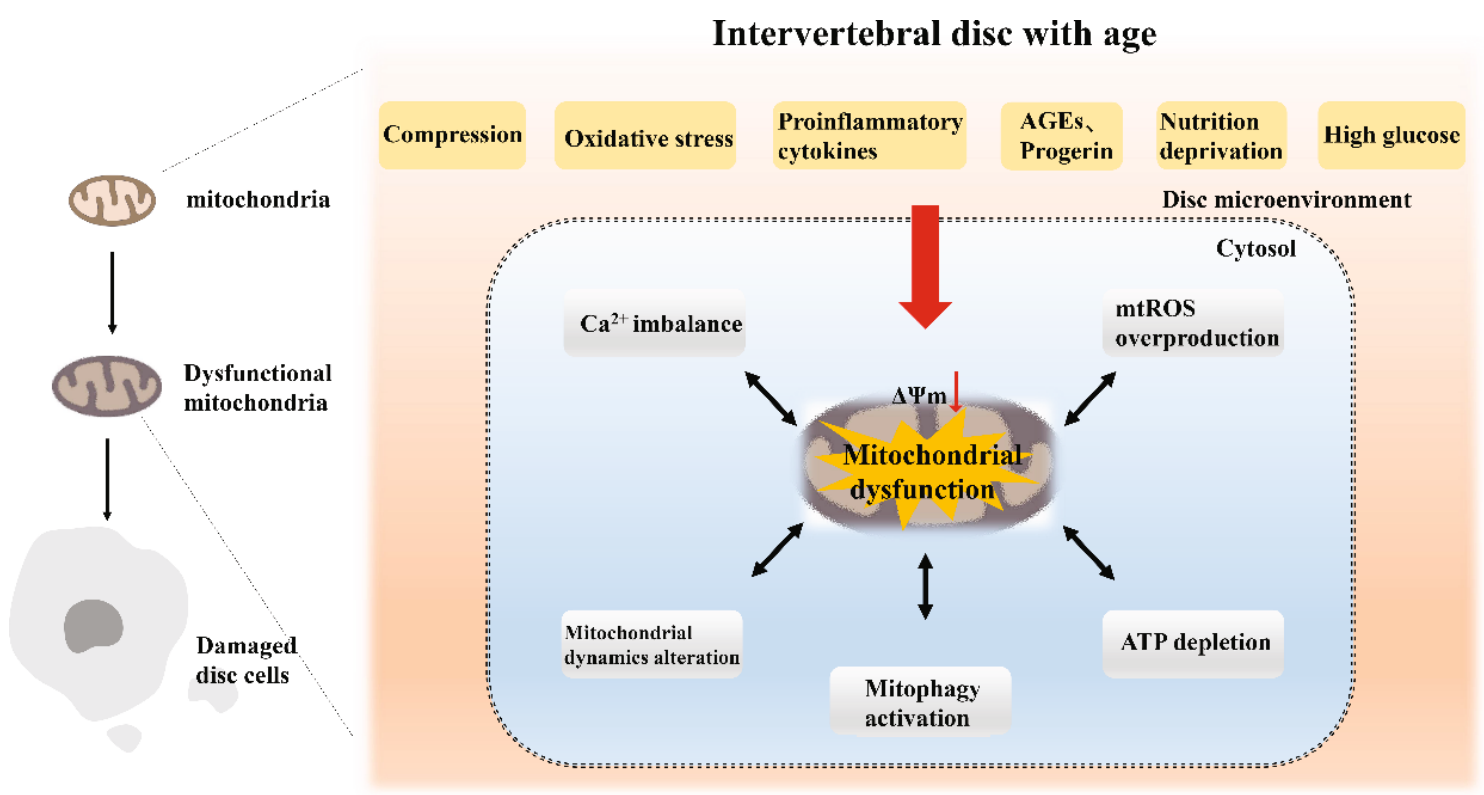

FIGURE 1: The occurrence of mitochondrial dysfunction in IVD cells. Mitochondrial dysfunction in IVD cells primarily originates from the effects of aberrant microenvironment within the disc including mechanical compression, proinflammatory cytokines, oxidative stress, nutrition deprivation, and high glucose. These pathological conditions induce ATP depletion, mtROS overproduction, Ca ${ }^{2+}$ imbalance, loss of $\Delta \psi \mathrm{m}$, and impairment of mitochondrial quality control, involved in multiple cellular events.

on phagophore [37]. Damaged mitochondria are engulfed by autophagosomes to form mitophagosomes, followed by the fusion with lysosomes for hydrolytic degradation [38]. With regard to Parkin-independent pathways, BNIP3, NIX, and FUNDC could directly bind the LC3 molecules decorating the autophagosome [39]. In addition, the mechanisms of mitophagy and mitochondrial dynamics are tightly related. For example, the Parkin-mediated ubiquitylation of MFN is able to promote mitochondria accessible for degradation and prevents fusion of damaged mitochondria. The dephosphorylation of FUNDC1 could enhance mitochondrial fission by the disassembly of OPA1 and increasing the interaction with DRP1 on the mitochondrial surface [39]. In physiological conditions, mitochondrial homeostasis could be achieved through these interactive processes. However, mitochondrial fusion and fission and mitophagy could be strongly dysregulated by various pathological stresses, such as $\Delta \psi \mathrm{m}$ collapse, oxidative stress, and nutrition deprivation, causing or accelerating mitochondrial dysfunction [40].

\section{The Roles of Mitochondrial Dysfunction in IVD Degeneration}

It has been shown an abnormal mitochondrial morphology with dark color, small cristae, and dense inclusion bodies, decreased mitochondrial mass, and reinforced mitochondrial respiration in human $\mathrm{AF}$ cells from degenerative discs [24]. The reduced mitochondrial respiration, $\Delta \psi \mathrm{m}$, and mitochondrial number were also found in NP and AF cells from aged rabbits compared with that from young rabbits [41]. These findings confirm the association between mitochondrial dysfunction and IVD degeneration. Importantly, the current evidence indicates that the pathophysiological char- acteristics of IVD degeneration primarily include oxidative stress, senescence and death of disc cell, and ECM degradation, all of which could be, at least partly, attributed to mitochondrial dysfunction. We will focus on the roles of mitochondrial dysfunction in the pathogenesis of IVD degeneration through these pathological processes (Figure 2).

3.1. Mitochondrial Dysfunction and Oxidative Stress. The accumulated evidence has shown that oxidative stress as a cause and/or consequence of mitochondrial dysfunction is one of the main drivers of aging and aging-related diseases [42]. Excessive ROS production has also been found in degenerative discs [43]. During the development of IVD degeneration, there exist various exogenous stimuli, such as mechanical loading, proinflammatory cytokines, high oxygen tension, and glucose stress in the microenvironment of IVD, inducing overproduction of ROS in disc cells [44]. Interestingly, mitochondrial dysfunction plays a core role in all of these processes. The compression, a type of mechanical stresses, is commonly used to mimic the pathological condition of IVDdegeneration. This stress promoted excessive mitochondrial ROS production accompanied by increased mPTP opening and decreased $\Delta \psi \mathrm{m}$ in NP cells $[25,45]$. In addition to the mechanical stress, proinflammatory cytokines, including interleukin- (IL-) $1 \beta$ and tumor necrosis factor-alpha (TNF- $\alpha$ ), increased in degenerative intervertebral disc, are also considered as critical contributors in the process of IVD degeneration [46, 47]. At the cellular level, these inflammatory mediators can trigger sustained $\mathrm{mPTP}$ opening, negatively alter mitochondrial membrane potential and ATP content, and cause aberrant mitochondrial fragmentation and swelling in NP cells, leading to mitochondrial dysfunction and ROS overproduction [46, 48, 49]. Previous 


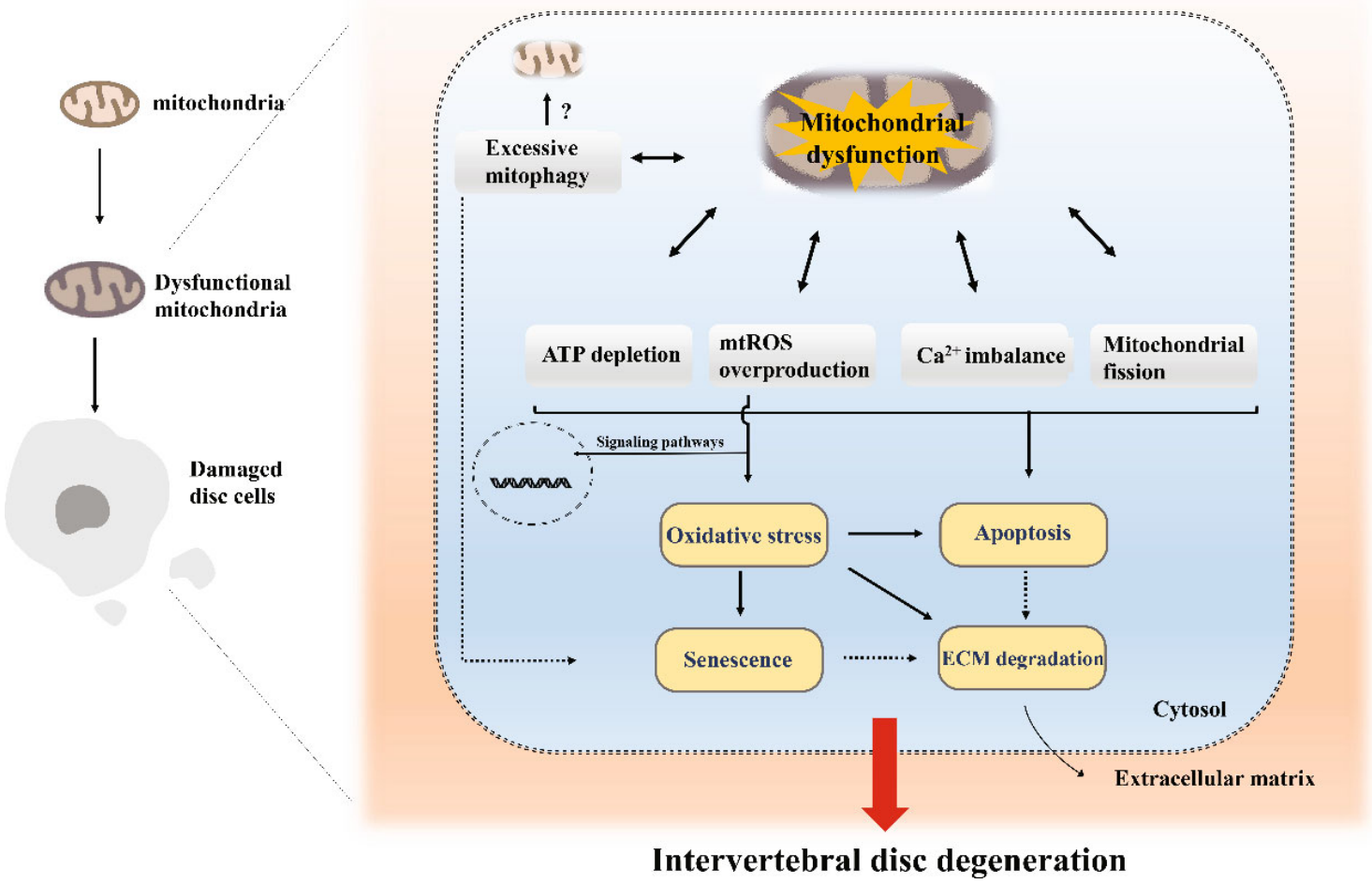

FIGURE 2: The role of mitochondrial dysfunction in the pathogenesis of IVD degeneration. The dysfunctional mitochondria could cause a series of cascade reactions, leading to increased oxidative stress, senescence, apoptosis, and ECM catabolism of disc cells, involved in the pathology of IVD degeneration.

studies have reported a higher incidence of degenerative disc diseases in patients with diabetes mellitus (DM) than in nondiabetic patients, and the accumulation of advanced glycation end products (AGEs) was potentially involved in DM and other age-related diseases $[50,51]$. Some researchers work on this topic and found that high glucose could also disrupt $\Delta \psi \mathrm{m}$ and enhance ROS generation in rat NP cells, $\mathrm{AF}$ cells, and CEP cells [27, 52-54]. In addition, it was reported that the accumulation of AGEs in NP tissues could induce mitochondrial dysfunction and an oxidative microenvironment, which was also closely correlated to IVD degeneration $[55,56]$. The above studies suggest the pathological stimulus and stresses in the microenvironment of IVD contribute to mitochondrion dysfunction and oxidative stress, implicated in the pathogenesis of IVD degeneration.

Mitochondria are not only the main cellular source of ROS; they are also susceptible to oxidative injury [57]. When stimulated by exogenous ROS, hydrogen peroxide, mitochondria in disc cells lose their ATP content, transmembrane potential, which induce the release of mtROS [58-60]. The ROS-induced mitochondrial dysfunction is able to further promote ROS generation, resulting in a feed-forward vicious cycle between ROS and mitochondria that causes a series of signaling cascades and oxidative damage [61].

3.2. Mitochondrial Dysfunction and Senescence. Cellular senescence is a permanent state of growth arrest characterized by the chronic elevated secretion of proinflammatory cytokines and matrix proteases, namely, senescence- associated secretory phenotype (SASP) [62]. Senescent cells have been found to be increased with age and degeneration of human IVD [63, 64]. Interestingly, senescence of IVD cells is one of the cascade events of mitochondrial dysfunction [65]. As reported, the mitochondria's role in senescent process was closely associated with overproduction of ROS that initials the related signaling networks to facilitate the senescent phenotype [66]. Specifically, a severe ROS production induced by $\mathrm{H}_{2} \mathrm{O}_{2}$ further activates the ATM-Chk2-p53p21- $\mathrm{Rb}$ pathway to enhance DNA damage and the accumulation of senescence-associated $\beta$-galactosidase, driving senescence of human NP cells $[58,67]$. In addition to NP cells, mitochondrial ROS-induced senescence was also found in human CEP cells, mediated by the p53-p21-Rb pathway [68]. Not only that, the impaired mitochondrial function and ROS generation is also involved in the senescence of IVD cells under other pathological conditions including proinflammatory cytokines, mechanical compression, agingrelated proteins, and high glucose [27, 48, 69, 70]. It was reported that the aberrant mitophagy caused by continuous compression could accelerate the senescence of NP cells, which we will discuss later [70]. Taking together, mitochondrial dysfunction is a major cause of cell senescence, and maintenance or recovery of mitochondrial function in IVD cells is important to mitigate cell senescence.

3.3. Mitochondrial Dysfunction and Apoptosis. The loss of IVD cells in the form of apoptosis is a key factor of IVD degeneration [71-73]. Mitochondrial-dependent apoptosis 
is one of the apoptotic pathways and most studied in the progression of IVD degeneration [74]. This apoptotic pathway requires mitochondrial outer membrane permeabilization (MOMP) driven by effector proapoptotic members of the B cell lymphoma 2 (BCL-2) family of proteins for the release of cytochrome $\mathrm{c}$ into the cytosol and further activating caspase (caspase3, 9) that leads to cell death [75]. The initiation of the above process heavily relies on the alteration of mitochondrial function, including elevated ROS production, the opening of mPTP, and mitochondrial fission, which have been confirmed in the apoptotic process of IVD cells induced by various pathological conditions. For example, $\mathrm{H}_{2} \mathrm{O}_{2}$ induced mitochondrial-dependent apoptosis through inhibiting the activity of complex III, decreasing the ATP levels and $\Delta \psi \mathrm{m}$ in AF cells [76]. IL- $1 \beta$ reduced $\mathrm{Bcl}-2 / \mathrm{Bax}$ ratio and enhanced cytochrome $\mathrm{c}$ released from the mitochondria to the cytosol, which proved mitochondrial-mediated apoptosis was induced in NP cells. The similar apoptotic process in disc cells was also found under the conditions of nutrient deprivation [77, 78], high glucose [52], and AGE accumulation [79]. In addition, compression elicited a timedependent mitochondrial dysfunction, evident by $\Delta \psi \mathrm{m}$ loss, mPTP opening, ATP depletion, and elevated mtROS, eventually leading to apoptosis [25] together with necroptosis in NP cells [80]. Chen et al. found that RIPK1 mediated this process of cell death during compression injury [80]. In short, these important pathogenic factors share similar mitochondrialdependent apoptotic mechanism, further confirming the core role of mitochondrial dysfunction in cell apoptosis and IVD degeneration. It should be noted that some molecules have recently been demonstrated to involve in mitochondrialdependent apoptosis in disc cells. Islet amyloid polypeptide (IAPP) is a polypeptide mainly participating in the regulation of glucose metabolism [81]; its expression was considerably decreased with the progression of IVD degeneration, and downregulated IAPP has a detrimental effect on AF cell survival through increasing cellular $\mathrm{Ca}^{2+}$ concentration, ROS, and apoptosis [82]. Acid-sensing ion channel 1a, a receptor of protons, also functions in CEP cells, allowing the influx of $\mathrm{Ca}^{2+}$ and triggering $\mathrm{Ca}^{2+}$-mediated apoptotic signals [83]. These molecules give a novel and wide insight into the mitochondrial pathway of apoptosis in degenerative disc cells.

\subsection{Mitochondrial Dysfunction and ECM Metabolism. ECM} degradation is a hallmark of IVD degeneration. The degeneration, driven by increased proteolysis of matrix components including aggrecan and type II collagen mediated by catabolic enzymes, like matrix metalloproteinases (MMPs) and a disintegrin and metalloproteinase with thrombospondin motifs (ADAMTS), is closely correlated to mitochondrial dysfunction in IVD cells [84]. On the one hand, anabolism of ECM requires ATP for providing energy [85] while mitochondrial dysfunction, showing ATP depletion, indeed reduces the anabolic process in IVD cells $[69,86]$. On the other hand, the mitochondrial-mediated ROS generation could significantly interrupt the metabolism of ECM. A number of studies showed reduced expression of aggrecan and type II collagen while increased expression of MMP13 in human and rat disc cells exposed to exogenous $\mathrm{H}_{2} \mathrm{O}_{2}$ $[67,87,88]$. Moreover, the ROS accumulation induced by mechanical compression and proinflammatory cytokines is associated with matrix degradation $[48,89]$. The excessive ROS not only is able to directly cause the oxidative damage of anabolic-related DNA and proteins but also acts as signaling messengers of NF-?B, nuclear factor, erythroid 2-like 2 (Nrf2)/HO-1, AKT, and mitogen-activated protein kinase (MAPK) pathways that affect the expression of ECM metabolic markers [67]. However, some pathological factors, especially for proinflammatory cytokines, could directly regulate the above signaling pathways, contributing to the inflammation-mediated ECM degradation in human and rat disc cells [90-92]. Taken all the results into account, although the molecular mechanism of ECM degradation is complicated, mitochondria, as a hub of multiple signal transduction, play a crucial role in this process. Impaired mitochondria are prone to drive the cascade reactions to promote matrix degradation and structure loss in IVD.

3.5. The Roles of Mitochondrial Energy Metabolism in IVD Degeneration. Maintenance of the ECM in the IVD is a high energy demanding process that requires glucose and oxygen consumption to produce ATP [22]. However, ATP content has been found decreased in degenerative disc under some pathological conditions $[69,76]$, and age-related decline in disc cellular bioenergetics has been considered as a contributor to the matrix degradation [93]. The previous study revealed a profound increase in glycolysis, altered mitochondrial morphology, and lower membrane potential but no change in mitochondrial respiration in AF cells from older rabbits [41]. In contrast, NP cells from older rabbits showed a significant decrease in OXPHOS without a concomitant increase in glycolysis, and it also exhibited a significant loss in maximum respiratory capacity and bioenergetic reserve [41], which may cause protein damage and cell death [94]. These limited mitochondrial capacities could hamper matrix repair or cellular stress responses. On the other hand, exogenous ATP could significantly promote ECM deposition and corresponding gene expression (aggrecan and type II collagen) in NP cells and AF cells in three-dimensional agarose culture [85].

These findings indicate that normal metabolism of energy is essential for ECM homeostasis, while age-related disc cell mitochondrial and bioenergetic changes might contribute to the loss of matrix homeostasis that underlay disc degeneration. However, it is important to note that the results of cell culture should be confirmed in vivo.

3.6. The Roles of Mitochondrial Dynamics and Mitophagy in IVD Degeneration. The recent evidence revealed that imbalanced mitochondrial dynamics and mitophagy are implicated in various age-related diseases including Alzheimer's disease, osteoarthritis, and IVD degeneration [45, 95-97]. Disturbed mitochondrial dynamics occurs in response to abnormal stresses and directly impacts mitochondrial function in disc cells. Recent studies have demonstrated that oxidative stress enhanced mitochondrial fission events and, meantime, impaired fusion events [59]. Additionally, 
compared to control cells, Mff and Fis1 protein levels were significantly upregulated, while MFN1, MFN2, and OPA1 protein levels were reduced in compression-treated NP cells [45]. Furthermore, blocking mitochondrial fission by mitochondrial division inhibitor 1 (mdivi-1) inhibited the elevated mitochondrial impairment, ROS generation, and apoptosis induced by compression treatment [45]. Mitochondrial fission also mediates programmed necrosis of NP cells. mdivi-1 and knockdown of DRP1 prevented the compression-induced programmed necrosis of NP cells by enhancing mitochondrial translocation of p53 and nuclear translocation of apoptosisinducing factor (AIF) [98]. MitoQ is a mitochondrialtargeted antioxidant that can rescue the oxidative damage and imbalance between mitochondrial fission and fusion induced by compression in human NP cells, while the beneficial effects of MitoQ could be partially repressed by an agonist of mitochondrial fission, FCCP [45]. Actually, mitochondrial fusion is also indispensable for autophagic flux and mitochondrial function in NP cells. Chen et al. found that MFN2 deficiency contributed to IVD degeneration due to its impairment on mitochondrial function and mitophagy. Furthermore, overexpression of MFN2 can attenuate oxidative damage of NP cells and the development of IVD degeneration in rats through the activation of PINK1/Parkin-mediated mitophagy [99]. Collectively, these studies demonstrate the importance of balanced mitochondrial dynamics in the maintenance of mitochondrial homeostasis and NP cell survival. The active mitochondrial fission promotes the separation of dysfunctional and even healthy mitochondria for their degradation through mitophagy. Several studies have shown that mitophagy is activated in degenerative disc cells [26, 83, 84]. Given that the initiation of mitophagy is commonly caused by loss of $\Delta \psi \mathrm{m}$, hypoxia condition, and overproduced ROS, it is not surprising that pathological factors, including oxidative stress, mechanical compression, and TNF- $\alpha$, are prone to induce mitophagy in disc cells $[49,70,100]$. So here comes the question, what is the role of mitophagy in mitochondrial dysfunction and the progression of IVD degeneration. On the one hand, mitophagy is considered a protective mechanism owing to its clearance of damaged mitochondria. Several laboratories revealed that inhibition of PINK1-Parkinmediated mitophagy aggravated TNF- $\alpha$ or oxidative stressstimulated mitochondrial dysfunction and apoptosis of disc cells, whereas the activated mitophagy induced by its activators such as melatonin and urolithin A could protect disc cells from apoptosis and retard IVD degeneration in vivo [88, 101]. On the other hand, it was reported that suppression of PINK1/Parkin-mediated mitophagy by a mitophagy inhibitor, cyclosporin A (CsA), or PINK1 knockdown attenuated the compression-induced senescence of NP cells [70]. Moreover, CsA exerted a protective role in oxidative stress-induced apoptosis of NP cells [102]. These results indicate continuous pathological stress might induce inordinate mitophagy with an excessive clearance of mitochondria which accelerates the damage of NP cells. Taken together, the role of mitophagy in cell survival and function is possibly dependent on the mitophagy level under the pathological conditions. The differential roles of mitophagy in degenerative disc cells and in vivo studies need further investigation.

\section{Therapeutic Strategies to Target Mitochondrial Dysfunction}

The emerging evidence implies that mitochondrial dysfunction plays a central role in the pathophysiology of IVD degeneration. The involvement of mitochondrial dysfunction in IVD degeneration also represents an attractive target for its therapies. Accordingly, following strategies focusing on the improvement of mitochondrial function, attenuation of mitochondrial dysfunction, and the elimination of damaged mitochondria might be beneficial for the IVD degeneration (Figure 3).

4.1. Targeting ROS. Numerous studies have shown that a wide range of antioxidants exerts a significant protective role in degenerative IVD cells and animal models. Nacetylcysteine (NAC) is a well-known ROS scavenger that can, directly and indirectly, enter the cell and react with glutamic acid and glycine to generate intracellular glutathione [103]. It has been widely reported that NAC attenuates premature senescence, autophagy, and apoptosis in disc cells by decreasing the level of mtROS [67, 104, 105]. Moreover, the oral administration of NAC suppresses oxidative stress, inflammation, and matrix catabolism in rat discs to mitigate IVD degeneration [105]. Hydrogen sulfide $\left(\mathrm{H}_{2} \mathrm{~S}\right)$, a gaseous signaling messenger, has attracted attention for its biological functions [106]. Several studies have shown that $\mathrm{H}_{2} \mathrm{~S}$ can readily scavenge ROS at higher rates than other classic antioxidants [107], and exogenous $\mathrm{H}_{2} \mathrm{~S}$ has demonstrated profound antioxidant and cytoprotective capabilities in physiologic systems exposed to ROS [108]. $\mathrm{H}_{2} \mathrm{~S}$ also plays a protective role in degenerative NP cells and in the rat model of IVD degeneration induced by needle puncture, and the underlying mechanism involves inhibitory effect of $\mathrm{H}_{2} \mathrm{~S}$ on mitochondrial dysfunction and endoplasmic reticulum stress in IL- $1 \beta$-induced NP cells [46]. Considering the powerful antioxidative property of $\mathrm{H}_{2} \mathrm{~S}$, it is likely that $\mathrm{H}_{2} \mathrm{~S}$ could repress the generation of ROS to protect against IVD degeneration.

In addition, some natural compounds with antioxidative properties have been demonstrated to be beneficial for mitochondrial function of IVD cells and IVD degeneration. Kinsenoside (3-(R)-3- $\beta$-D-glucopyranosyloxybutanolide) is a bioactive monomer extracted from the Anoectochilus roxburghii [109]. This compound exerts its therapeutic potential through alleviating IVD degeneration in a punctureinduced model and protecting NP cells against mitochondrial dysfunction, senescence, and apoptosis under oxidative stress in an Nrf2-dependent way [110]. In line with kinsenoside, other natural compounds, such as epigallocatechin 3gallate (EGCG), resveratrol, icariin, and polydatin, also have shown promising effects at cellular levels and/or animal models of this disease $[58,111-113]$. These agents propose more choices for new drug development. Recently, antioxidant compounds incorporating ubiquinone (MitoQ) or TEMPO (Mito-TEMPO) specifically targeted to mitochondria have been successively used against mitochondrial dysfunction [114]. MitoQ consists of coenzyme Q10 and a TPP cation that can easily accumulate in the mitochondria, 


\section{Intervertebral disc with age}

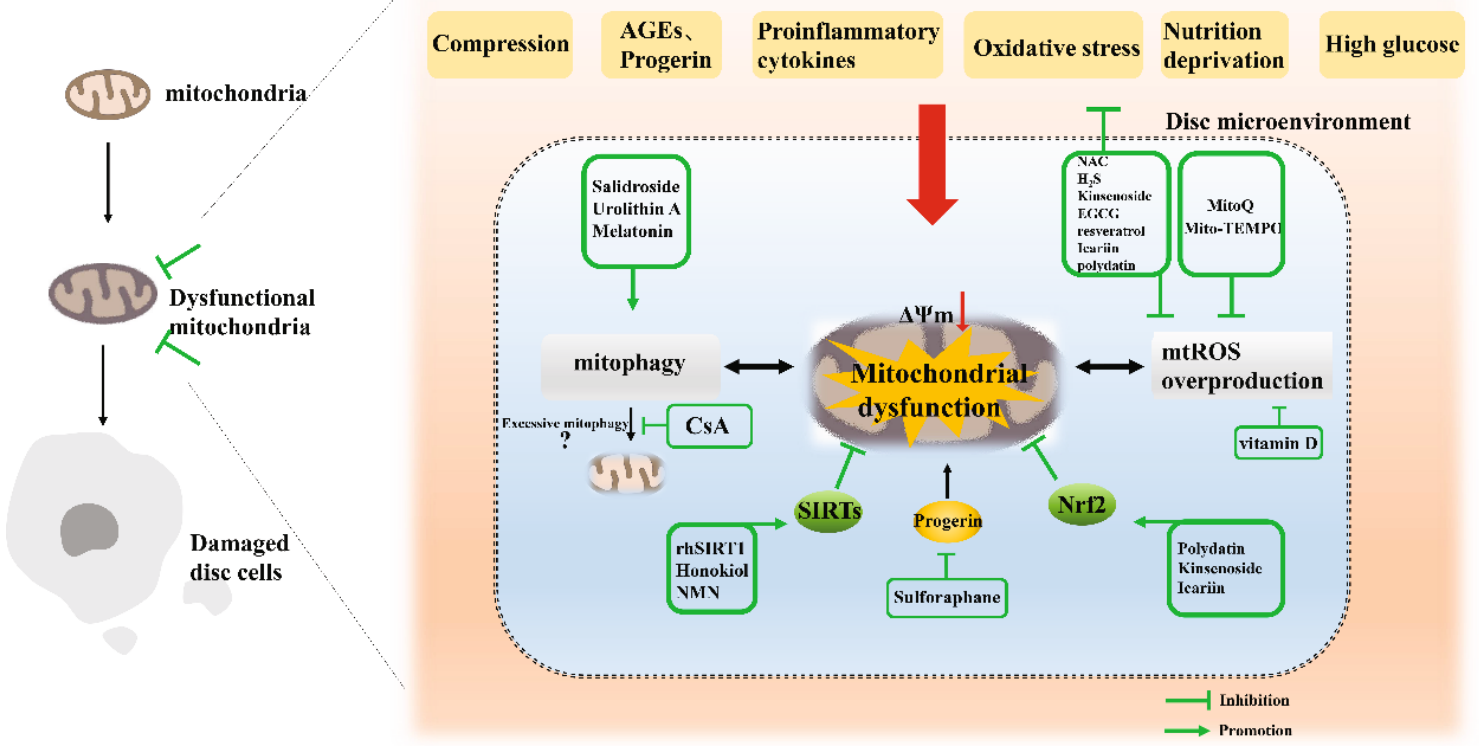

Figure 3: The therapies that target mitochondrial dysfunction. According to the role of mitochondrial dysfunction in IVD degeneration, multiple therapeutic agents that function on the improvement of mitochondrial function, attenuation of mitochondrial dysfunction, and the elimination of damaged mitochondria might be beneficial for the IVD degeneration.

thereby making it more powerful in preventing mitochondrial oxidative damage than untargeted antioxidants [115]. Actually, MitoQ restores mitochondrial function, eradicates oxidative insults, and promotes the survival of human NP cells under pathological condition [45], suggesting that MitoQ might serve as an effective therapeutic agent for IVD degeneration. Like MitoQ, Mito-TEMPO is also a mitochondrial-targeted antioxidant and protects NP cells against oxidative damage [60]. MitoVitE is another mitochondrial-targeted antioxidant. However, there is no evidence about its effects on degenerative IVD. Given its dramatic properties of antioxidation and antiapoptosis in multiple age-related diseases [116, 117], MitoVitE also deserves to be further studied and developed as a potential drug in IVD degeneration.

4.2. Targeting Mitophagy. Regulating mitophagy presents as an effective approach due to the involvement of impaired mitophagy in the development of IVD degeneration. Increasing Parkin-mediated mitophagy by using salidroside has been shown to eliminate impaired mitochondria and promote the survival of NP cells under TNF- $\alpha$ treatment. Salidroside also ameliorated the apoptosis of NP cells and the progression of IVD degeneration [49]. Urolithin A and melatonin have been widely studied in various diseases owing to their dramatic role in inducing mitophagy $[118,119]$. Therefore, it is not surprising that both compounds could effectively alleviate cellular oxidative damage and the progression of puncture-induced IVD degeneration in rats $[88,101]$. These evidences indicate Parkin-mediated mitophagy as a potential therapeutic target for IVD degeneration.

As mentioned above, there might exist dual roles of mitophagy with the development of IVD degeneration. Several groups have found that the suppression of excessive mitophagy displays a positive effect in preclinical experiments. $\mathrm{Xu}$ et al. found that oxidative stress induced apoptotic cell death via mitophagy while inhibiting mitophagy by CsA protected AF cells against apoptosis [102]. And they further found that repression of mitophagy could also alleviate the apoptosis of NP cells [120]. In addition, another study reported that CSA and PINK1 knockdown inhibited PINK1/Parkin-mediated mitophagy, thereby rescuing the senescence of NP cells under the treatment of compression [70]. These results imply that the excessive mitophagy in disc cells is deleterious and might be a target for therapeutic intervention. However, the changing roles of mitophagy during the development of IVD degeneration are not fully clarified. For better therapeutic applications that target mitophagy, it is necessary to elaborate the definite role of mitophagy in IVD at different pathophysiological stages and proper time window of therapeutic intervention in further studies.

4.3. Targeting Molecules and Signaling. Mitochondrial homeostasis is precisely regulated by signaling networks, among which some molecules present therapeutic potential as pharmacologic targets. As previously described, the level of progerin is elevated in degenerated human NP tissues and the accumulated progerin is involved in the pathogenesis of IVD degeneration. Sulforaphane was demonstrated to show a remarkable ability to modulate the progerin level and mitochondrial homeostasis, and consequently, this compound alleviated progerin-induced IVD degeneration [69]. Previous studies have exhibited that vitamin D via its receptor (VDR) can effectively scavenge free radicals to protect cell mitochondria [121]. A recent study showed that the expression level of VDR was significantly reduced in the disc of 
aged rats and severe degenerative discs. However, activation of VDR by $1,25(\mathrm{OH})_{2} \mathrm{D}_{3}$ successfully preserved mitochondrial function and ameliorated oxidative stress-induced apoptosis of AF cells, providing a novel insight into the treatment of IVD degeneration [76].

Growing shreds of evidence have demonstrated that sirtuins (SIRTs) are essential for every aspect of mitochondrial biology, including energy metabolism, ROS detoxification, mitochondrial dynamics, and mitophagy [122]. Miyazaki et al. reported that administration of recombinant human SIRT1 (rhSIRT1) exhibited a protective effect on nutrient deprivation-induced mitochondrial-dependent apoptosis in human NP cells, suggesting that rhSIRT1 may be a potent treatment agent for IVD diseases [77]. In addition, activated SIRT3 through adenosine monophosphate-activated protein kinase (AMPK) and peroxisome proliferator-activated receptor- $\gamma$ coactivator $1 \alpha$ (PGC- $1 \alpha$ ) pathway enhances mitochondrial function, mitochondrial antioxidant capacity, and mitochondrial dynamics, thereby attenuating AGEs or oxidative stress-induced apoptosis and senescence of NP cells and IVD degeneration in vivo [59, 79]. It is noteworthy that these functions of SIRT3 can be promoted by honokiol and nicotinamide mononucleotide (NMN) $[59,79]$, which provide available activators of SIRT3 and make SIRT3 possible to delay IVD degeneration as a target. Nrf2 is a master transcription factor that regulates cellular redox state and mitochondrial function [123]. In a mouse model of IVD degeneration, the Nrf2-knockout mice showed more severe degenerative changes in IVD than those in wild-type mice [124], and knockdown of $\mathrm{Nrf} 2$ accelerated $\mathrm{H}_{2} \mathrm{O}_{2}$-induced damage of mitochondria and CEP cell [60]. Furthermore, some phytochemicals including polydatin, kinsenoside, and icariin were proved to protect IVD cells from mitochondrial dysfunction and apoptosis through activating Nrf2 signaling $[48,110,113]$. These findings confirm the essential role of Nrf2 signaling in cellular homeostasis of the disc and highlight it as an effective therapeutic target in the management of IVD degeneration.

\section{Conclusion}

Mitochondrial functions, mainly including energy metabolism, redox, and calcium regulation, are altered in the degenerative disc. Mitochondrial dysfunction in IVD cells primarily originates from the effects of aberrant microenvironment within the disc including mechanical compression, proinflammatory cytokines, oxidative stress, nutrition deprivation, and high glucose. The dysfunctional mitochondria along with impaired mitochondrial quality control are prone to cause a series of cascade reactions, leading to ROS overproduction, senescence, apoptosis, and ECM catabolism of disc cells, involved in the pathology of IVD degeneration. Based on this, the therapies that target the mtROS, mitophagy, and relative molecules and signaling are promising for IVD degeneration, which extensive evidence has been demonstrated in preclinical experiments. However, these therapeutic approaches were studied in a certain in vitro condition or animal model of IVD degeneration that cannot completely represent the pathophysiology of IVD degenera- tion in humans. Moreover, some topics, such as the role of mitophagy in the degenerative disc, are still controversial. For the earlier and better clinical intervention by targeting mitochondrial dysfunction, it will be necessary to verify these therapeutic approaches in more animal models and clinical studies.

\section{Abbreviations}

IVD: Intervertebral disc

NP: $\quad$ Nucleus pulposus

GAGs: Glycosaminoglycans

AF: Annulus fibrosus

CEP: $\quad$ Cartilaginous endplates

ECM: $\quad$ Extracellular matrix

LBP: $\quad$ Lower back pain

MMP: Mitochondrial transmembrane potential

mtDNA: Mitochondrial DNA

ROS: $\quad$ Reactive oxygen species

TCA: Tricarboxylic acid

OXPHOS: Oxidative phosphorylation

ETC: $\quad$ Electron transport chain

IMM: Inner mitochondrial membrane

$\Delta \psi \mathrm{m}: \quad$ Mitochondrial membrane potential

$\mathrm{O}_{2}^{-}$: $\quad$ Superoxide anion

$\mathrm{H}_{2} \mathrm{O}_{2}$ : Hydrogen peroxide

$\mathrm{OH}-$ : $\quad$ Hydroxyl radical

mtROS: Mitochondrial-dependent ROS

$\mathrm{Ca}^{2+}: \quad$ Calcium

mPTP: $\quad$ Mitochondrial permeability transition pore

DRP1: Dynamin-related protein 1

Mff: $\quad$ Mitochondrial fission factor

Fis 1: $\quad$ Fission 1

OMM: $\quad$ Outer mitochondrial membrane

MFN1: $\quad$ Mitofusin 1

MFN2: $\quad$ Mitofusin 2

OPA1: $\quad$ Optic atrophy 1

mdivi-1: Mitochondrial division inhibitor 1

Parkin: $\quad$ Parkin RBR E3 ubiquitin-protein ligase

IL- $1 \beta$ : Interleukin- $1 \beta$

TNF- $\alpha$ : Tumor necrosis factor-alpha

DM: $\quad$ Diabetes mellitus

AGEs: $\quad$ Advanced glycation end products

SASP: $\quad$ Senescence-associated secretory phenotype

MOMP: Mitochondrial outer membrane permeabilization

BCL-2: $\quad$ B cell lymphoma 2

MMPs: $\quad$ Matrix metalloproteinases

ADAMTS: A disintegrin and metalloproteinase with thrombospondin motifs

Nrf2: $\quad$ Nuclear factor, erythroid 2-like 2

MAPKs: Mitogen-activated protein kinases

AIF: Apoptosis-inducing factor

CsA: Cyclosporin A

NAC: $\quad$ N-acetylcysteine

H2S: Hydrogen sulfide

EGCG: Epigallocatechin 3-gallate

MitoQ: Mitoquinone

VDR: Vitamin D receptor 


$\begin{array}{ll}\text { SIRTs: } & \text { Sirtuins } \\ \text { rhSIRT1: } & \text { Recombinant human SIRT1 } \\ \text { AMPK: } & \begin{array}{l}\text { Adenosine monophosphate-activated protein } \\ \text { kinase }\end{array} \\ \text { PGC-1 } \alpha: & \begin{array}{l}\text { Peroxisome proliferator-activated receptor- } \gamma \\ \text { coactivator } 1 \alpha\end{array} \\ \text { NMN: } & \text { Nicotinamide mononucleotide. }\end{array}$

\section{Conflicts of Interest}

The authors declare that there is no conflict of interest regarding the publication of this article.

\section{Authors' Contributions}

Lin Jin worked on design and conception of this review and drafted the paper. Danni Li and Fenghua Tao contributed to revise the paper. Danni Li worked on the visualization, and all authors approved the final version of the manuscript.

\section{References}

[1] P. P. A. Vergroesen, I. Kingma, K. S. Emanuel et al., "Mechanics and biology in intervertebral disc degeneration: a vicious circle," Osteoarthritis and Cartilage, vol. 23, no. 7, pp. 1057-1070, 2015.

[2] G. Fontana, E. See, and A. Pandit, "Current trends in biologics delivery to restore intervertebral disc anabolism," Advanced Drug Delivery Reviews, vol. 84, pp. 146-158, 2015.

[3] M. V. Risbud, Z. R. Schoepflin, F. Mwale et al., "Defining the phenotype of young healthy nucleus pulposus cells: recommendations of the spine research interest group at the 2014 annual ORS meeting," Journal of Orthopaedic Research, vol. 33, no. 3, pp. 283-293, 2015.

[4] C. Maher, M. Underwood, and R. Buchbinder, "Non-specific low back pain," The Lancet, vol. 389, no. 10070, pp. 736-747, 2017.

[5] N. Henry, J. Clouet, J. Le Bideau, C. Le Visage, and J. Guicheux, "Innovative strategies for intervertebral disc regenerative medicine: from cell therapies to multiscale delivery systems," Biotechnology Advances, vol. 36, no. 1, pp. 281294, 2018.

[6] C. J. L. Murray, T. Vos, R. Lozano et al., "Disability-adjusted life years (DALYs) for 291 diseases and injuries in 21 regions, 1990-2010: a systematic analysis for the global burden of disease study 2010," The Lancet, vol. 380, no. 9859, pp. 21972223, 2012.

[7] G. Livshits, M. Popham, I. Malkin et al., "Lumbar disc degeneration and genetic factors are the main risk factors for low back pain in women: the UK twin spine study," Annals of the Rheumatic Diseases, vol. 70, no. 10, pp. 1740-1745, 2011.

[8] T. Oichi, Y. Taniguchi, Y. Oshima, S. Tanaka, and T. Saito, "Pathomechanism of intervertebral disc degeneration," JOR Spine, vol. 3, no. 1, article e1076, 2020.

[9] A. Elfering, N. Semmer, D. Birkhofer, M. Zanetti, J. Hodler, and N. Boos, "Young Investigator Award 2001 Winner: risk factors for lumbar disc Degeneration," Spine, vol. 27, no. 2, pp. 125-134, 2002.

[10] J. Clouet, C. Vinatier, C. Merceron et al., “The intervertebral disc: from pathophysiology to tissue engineering," Joint, Bone, Spine, vol. 76, no. 6, pp. 614-618, 2009.
[11] G. S. Shadel and T. L. Horvath, "Mitochondrial ROS signaling in organismal homeostasis," Cell, vol. 163, no. 3, pp. 560-569, 2015.

[12] M. Giacomello, I. Drago, P. Pizzo, and T. Pozzan, "Mitochondrial Ca2+ as a key regulator of cell life and death," Cell Death \& Differentiation, vol. 14, no. 7, pp. 1267-1274, 2007.

[13] R. J. Youle and A. M. van der Bliek, "Mitochondrial fission, fusion, and stress," Science, vol. 337, no. 6098, pp. 10621065, 2012.

[14] S. Pickles, P. Vigié, and R. J. Youle, "Mitophagy and quality control mechanisms in mitochondrial maintenance," Current Biology: CB, vol. 28, no. 4, pp. R170-R185, 2018.

[15] M. T. Lin and M. Flint Beal, "Mitochondrial dysfunction and oxidative stress in neurodegenerative diseases," Nature, vol. 443, no. 7113, pp. 787-795, 2006.

[16] W.-X. Zong, J. D. Rabinowitz, and E. White, "Mitochondria and cancer," Molecular Cell, vol. 61, no. 5, pp. 667-676, 2016.

[17] J. Chow, J. Rahman, J. C. Achermann, M. T. Dattani, and S. Rahman, "Mitochondrial disease and endocrine dysfunction," Nature Reviews Endocrinology, vol. 13, no. 2, pp. 92104, 2017.

[18] D. R. Green, L. Galluzzi, and G. Kroemer, "Mitochondria and the autophagy-inflammation-cell death axis in organismal aging," Science (New York, N.Y.), vol. 333, no. 6046, pp. 1109-1112, 2011.

[19] D. F. Wilson, "Oxidative phosphorylation: regulation and role in cellular and tissue metabolism," The Journal of Physiology, vol. 595, no. 23, pp. 7023-7038, 2017.

[20] P. R. Rich and A. Maréchal, "The mitochondrial respiratory chain," Essays in Biochemistry, vol. 47, pp. 1-23, 2010.

[21] S. R. S. Bibby, D. A. Jones, R. M. Ripley, and J. P. G. Urban, "Metabolism of the intervertebral disc: effects of low levels of oxygen, glucose, and $\mathrm{pH}$ on rates of energy metabolism of bovine nucleus pulposus cells," Spine, vol. 30, no. 5, pp. 487-496, 2005.

[22] J. C. Salvatierra, T. Y. Yuan, H. Fernando et al., "Difference in energy metabolism of annulus fibrosus and nucleus pulposus cells of the intervertebral disc," Cellular and Molecular Bioengineering, vol. 4, no. 2, pp. 302-310, 2011.

[23] W. Dröge, "Free radicals in the physiological control of cell function," Physiological Reviews, vol. 82, no. 1, pp. 47-95, 2002.

[24] H. E. Gruber, J. A. Watts, F. E. Riley, F. Mary-Beth, H. James Norton, and E. N. Hanley Jr., "Mitochondrial bioenergetics, mass, and morphology are altered in cells of the degenerating human annulus," Journal of Orthopaedic Research, vol. 31, no. 8, pp. 1270-1275, 2013.

[25] F. Ding, Z.-W. Shao, S.-H. Yang, Q. Wu, F. Gao, and L.M. Xiong, "Role of mitochondrial pathway in compressioninduced apoptosis of nucleus pulposus cells," Apoptosis, vol. 17, no. 6, pp. 579-590, 2012.

[26] L. A. Nasto, A. R. Robinson, K. Ngo et al., "Mitochondrialderived reactive oxygen species (ROS) play a causal role in aging-related intervertebral disc degeneration," Journal of Orthopaedic Research, vol. 31, no. 7, pp. 1150-1157, 2013.

[27] J.-S. Park, J.-B. Park, I.-J. Park, and E.-Y. Park, “Accelerated premature stress-induced senescence of young annulus fibrosus cells of rats by high glucose-induced oxidative stress," International Orthopaedics, vol. 38, no. 6, pp. 1311-1320, 2014 . 
[28] R. B. Hamanaka and N. S. Chandel, "Mitochondrial reactive oxygen species regulate cellular signaling and dictate biological outcomes," Trends in Biochemical Sciences, vol. 35, no. 9, pp. 505-513, 2010.

[29] T. Finkel and N. J. Holbrook, "Oxidants, oxidative stress and the biology of ageing," Nature, vol. 408, no. 6809, pp. 239247, 2000.

[30] K. Kalani, S. F. Yan, and S. S. Du Yan, "Mitochondrial permeability transition pore: a potential drug target for neurodegeneration," Drug Discovery Today, vol. 23, no. 12, pp. 1983-1989, 2018.

[31] A. P. Halestrap, "What is the mitochondrial permeability transition pore?," Journal of Molecular and Cellular Cardiology, vol. 46, no. 6, pp. 821-831, 2009.

[32] L. Tilokani, S. Nagashima, V. Paupe, and J. Prudent, "Mitochondrial dynamics: overview of molecular mechanisms," Essays in Biochemistry, vol. 62, no. 3, pp. 341-360, 2018.

[33] L. D. Osellame, A. P. Singh, D. A. Stroud et al., "Cooperative and independent roles of the Drp 1 adaptors Mff, MiD49 and MiD51 in mitochondrial fission," Journal of Cell Science, vol. 129, no. 11, pp. 2170-2181, 2016.

[34] A. Santel and M. T. Fuller, "Control of mitochondrial morphology by a human mitofusin," Journal of Cell Science, vol. 114, no. 5, p. 867, 2001.

[35] T. Wai and T. Langer, "Mitochondrial dynamics and metabolic regulation," Trends in Endocrinology \& Metabolism, vol. 27, no. 2, pp. 105-117, 2016.

[36] B. Bingol and M. Sheng, "Mechanisms of mitophagy: PINK1, Parkin, USP30 and beyond," Free Radical Biology and Medicine, vol. 100, pp. 210-222, 2016.

[37] T. N. Nguyen, B. S. Padman, and M. Lazarou, "Deciphering the molecular signals of PINK1/parkin mitophagy," Trends in Cell Biology, vol. 26, no. 10, pp. 733-744, 2016.

[38] T. Kanki, D. J. Klionsky, and K. Okamoto, "Mitochondria autophagy in yeast," Antioxidants \& Redox Signaling, vol. 14, no. 10, pp. 1989-2001, 2011.

[39] K. Palikaras, E. Lionaki, and N. Tavernarakis, "Mechanisms of mitophagy in cellular homeostasis, physiology and pathology," Nature Cell Biology, vol. 20, no. 9, pp. 1013-1022, 2018.

[40] C. Guo, G. Kroemer, and O. Kepp, "Mitophagy: an emerging role in aging and age-associated diseases," Frontiers in Cell and Developmental Biology, vol. 8, p. 200, 2020.

[41] R. Hartman, P. Patil, R. Tisherman et al., "Age-dependent changes in intervertebral disc cell mitochondria and bioenergetics," European Cells and Materials, vol. 36, pp. 171-183, 2018.

[42] D. A. Pulliam, A. Bhattacharya, and H. Van Remmen, "Mitochondrial dysfunction in aging and longevity: a causal or protective role?," Antioxidants \& Redox Signaling, vol. 19, no. 12, pp. 1373-1387, 2013.

[43] K.-W. Kim, H.-N. Chung, K.-Y. Ha, J.-S. Lee, and Y.-Y. Kim, "Senescence mechanisms of nucleus pulposus chondrocytes in human intervertebral discs," The Spine Journal, vol. 9, no. 8, pp. 658-666, 2009.

[44] C. Feng, M. Yang, M. Lan et al., "ROS: crucial intermediators in the pathogenesis of intervertebral disc degeneration," Oxidative Medicine and Cellular Longevity, vol. 2017, Article ID 5601593, 12 pages, 2017.

[45] K. Liang, S. Liu, J. Li, Y. Tian, Y. Xue, and X. Liu, "The mitochondria-targeted anti-oxidant Mito $\mathrm{Q}$ protects against intervertebral disc degeneration by ameliorating mitochon- drial dysfunction and redox imbalance," Cell Proliferation, vol. 53, no. 3, article e12779, 2020.

[46] D. Xu, H. Jin, J. Wen et al., "Hydrogen sulfide protects against endoplasmic reticulum stress and mitochondrial injury in nucleus pulposus cells and ameliorates intervertebral disc degeneration," Pharmacological Research, vol. 117, pp. 357$369,2017$.

[47] M. V. Risbud and I. M. Shapiro, "Role of cytokines in intervertebral disc degeneration: pain and disc content," Nature Reviews Rheumatology, vol. 10, no. 1, pp. 44-56, 2014.

[48] J. Wang, C. Huang, Z. Lin et al., "Polydatin suppresses nucleus pulposus cell senescence, promotes matrix homeostasis and attenuates intervertebral disc degeneration in rats," Journal of Cellular and Molecular Medicine, vol. 22, no. 11, pp. 5720-5731, 2018.

[49] Z. Zhang, T. Xu, J. Chen et al., "Parkin-mediated mitophagy as a potential therapeutic target for intervertebral disc degeneration," Cell Death \& Disease, vol. 9, no. 10, p. 980, 2018.

[50] M. Teraguchi, N. Yoshimura, H. Hashizume et al., "Progression, incidence, and risk factors for intervertebral disc degeneration in a longitudinal population-based cohort: the Wakayama spine study," Osteoarthritis and Cartilage, vol. 25, no. 7, pp. 1122-1131, 2017.

[51] S.-Y. Goh and M. E. Cooper, "The role of advanced glycation end products in progression and complications of diabetes," The Journal of Clinical Endocrinology \& Metabolism, vol. 93, no. 4, pp. 1143-1152, 2008.

[52] Z. Jiang, W. Lu, Q. Zeng, D. Li, L. Ding, and W. Jingping, "High glucose-induced excessive reactive oxygen species promote apoptosis through mitochondrial damage in rat cartilage endplate cells," Journal of Orthopaedic Research, vol. 36, no. 9, pp. 2476-2483, 2018.

[53] E.-Y. Park and J.-B. Park, "High glucose-induced oxidative stress promotes autophagy through mitochondrial damage in rat notochordal cells," International Orthopaedics, vol. 37, no. 12, pp. 2507-2514, 2013.

[54] M.-B. Guo, D.-C. Wang, H.-F. Liu et al., "Lupeol against high-glucose-induced apoptosis via enhancing the antioxidative stress in rabbit nucleus pulposus cells," European Spine Journal, vol. 27, no. 10, pp. 2609-2620, 2018.

[55] Y. Song, Y. Wang, Y. Zhang et al., "Advanced glycation end products regulate anabolic and catabolic activities via NLRP3-inflammasome activation in human nucleus pulposus cells," Journal of Cellular and Molecular Medicine, vol. 21, no. 7, pp. 1373-1387, 2017.

[56] T.-T. Tsai, N. Y.-J. Ho, Y.-T. Lin et al., "Advanced glycation end products in degenerative nucleus pulposus with diabetes," Journal of Orthopaedic Research, vol. 32, no. 2, pp. 238-244, 2014.

[57] H.-M. Ni, J. A. Williams, and W.-X. Ding, "Mitochondrial dynamics and mitochondrial quality control," Redox Biology, vol. 4, pp. 6-13, 2015.

[58] O. Krupkova, J. Handa, M. Hlavna et al., "The natural polyphenol epigallocatechin gallate protects intervertebral disc cells from oxidative stress," Oxidative Medicine and Cellular Longevity, vol. 2016, Article ID 7031397, 17 pages, 2016.

[59] J. Wang, M. Nisar, C. Huang et al., "Small molecule natural compound agonist of SIRT3 as a therapeutic target for the treatment of intervertebral disc degeneration," Experimental \& Molecular Medicine, vol. 50, no. 11, pp. 1-14, 2018. 
[60] K. Liang, S. Liu, J. Li, Y. Tian, Y. Xue, and X. Liu, "Parkin and Nrf 2 prevent oxidative stress-induced apoptosis in intervertebral endplate chondrocytes via inducing mitophagy and anti-oxidant defenses," Life Sciences, vol. 243, p. 117244, 2020.

[61] J. W. Chen, B. B. Ni, B. Li, Y. H. Yang, S. D. Jiang, and L. S. Jiang, "The responses of autophagy and apoptosis to oxidative stress in nucleus pulposus cells: implications for disc degeneration," Cellular Physiology and Biochemistry, vol. 34, no. 4, pp. 1175-1189, 2014.

[62] C. D. Wiley and J. Campisi, "From ancient pathways to aging cells-connecting metabolism and cellular senescence," Cell Metabolism, vol. 23, no. 6, pp. 1013-1021, 2016.

[63] L. Maitre, C. Lyn, A. J. Freemont, and J. A. Hoyland, "Accelerated cellular senescence in degenerate intervertebral discs: a possible role in the pathogenesis of intervertebral disc degeneration," Arthritis Research \& Therapy, vol. 9, no. 3, p. R45, 2007.

[64] H. E. Gruber, J. A. Ingram, D. E. Davis, and E. N. Hanley, "Increased cell senescence is associated with decreased cell proliferation in vivo in the degenerating human annulus," The Spine Journal, vol. 9, no. 3, pp. 210-215, 2009.

[65] P. Patil, M. Falabella, A. Saeed et al., "Oxidative stressinduced senescence markedly increases disc cell bioenergetics," Mechanisms of Ageing And Development, vol. 180, pp. 97-106, 2019.

[66] J. F. Passos, G. Nelson, C. Wang et al., "Feedback between P 21 and reactive oxygen production is necessary for cell senescence," Molecular Systems Biology, vol. 6, no. 1, p. 347, 2010.

[67] A. Dimozi, E. Mavrogonatou, A. Sklirou, and D. Kletsas, "Oxidative stress inhibits the proliferation, induces premature senescence and promotes a catabolic phenotype in human nucleus pulposus intervertebral disc cells," European Cells \& Materials, vol. 30, pp. 89-103, 2015.

[68] N. Zhou, X. Lin, W. Dong et al., "SIRT1 alleviates senescence of degenerative human intervertebral disc cartilage endoplate cells via the p53/p21 pathway," Scientific Reports, vol. 6 , no. 1, p. 22628,2016

[69] X. Xu, D. Wang, C. Zheng et al., "Progerin accumulation in nucleus pulposus cells impairs mitochondrial function and induces intervertebral disc degeneration and therapeutic effects of sulforaphane," Theranostics, vol. 9, no. 8, pp. 2252-2267, 2019.

[70] D. Huang, Y. Peng, Z. Li et al., "Compression-induced senescence of nucleus pulposus cells by promoting mitophagy activation via the PINK1/PARKIN pathway," Journal of Cellular and Molecular Medicine, vol. 24, no. 10, pp. 5850-5864, 2020.

[71] C.-Q. Zhao, Y.-H. Zhang, S.-D. Jiang, L.-S. Jiang, and L.Y. Dai, "Both endoplasmic reticulum and mitochondria are involved in disc cell apoptosis and intervertebral disc degeneration in rats," Age (Dordrecht, Netherlands), vol. 32, no. 2, pp. 161-177, 2010.

[72] H. Sudo and A. Minami, "Caspase 3 as a therapeutic target for regulation of intervertebral disc degeneration in rabbits," Arthritis and Rheumatism, vol. 63, no. 6, pp. 1648-1657, 2011.

[73] S. Yang, F. Zhang, J. Ma, and W. Ding, "Intervertebral disc ageing and degeneration: the antiapoptotic effect of oestrogen," Ageing Research Reviews, vol. 57, p. 100978, 2020.

[74] H. Wang, H. Liu, Z.-M. Zheng et al., "Role of death receptor, mitochondrial and endoplasmic reticulum pathways in dif- ferent stages of degenerative human lumbar disc," Apoptosis, vol. 16, no. 10, pp. 990-1003, 2011.

[75] S. W. G. Tait and D. R. Green, "Mitochondria and cell death: outer membrane permeabilization and beyond," Nature Reviews Molecular Cell Biology, vol. 11, no. 9, pp. 621-632, 2010.

[76] T. Tong, Z. Liu, H. Zhang et al., "Age-dependent expression of the vitamin $D$ receptor and the protective effect of vitamin D receptor activation on $\mathrm{H} 2 \mathrm{O} 2$-induced apoptosis in rat intervertebral disc cells," The Journal of Steroid Biochemistry and Molecular Biology, vol. 190, pp. 126-138, 2019.

[77] S. Miyazaki, K. Kakutani, T. Yurube et al., "Recombinant human SIRT1 protects against nutrient deprivation-induced mitochondrial apoptosis through autophagy induction in human intervertebral disc nucleus pulposus cells," Arthritis Research \& Therapy, vol. 17, no. 1, p. 253, 2015.

[78] J. Liu, J. Wang, and Y. Zhou, "Upregulation of BNIP3 and translocation to mitochondria in nutrition deprivation induced apoptosis in nucleus pulposus cells," Joint, Bone, Spine, vol. 79, no. 2, pp. 186-191, 2012.

[79] Y. Song, S. Li, W. Geng et al., "Sirtuin 3-dependent mitochondrial redox homeostasis protects against AGEs- induced intervertebral disc degeneration," Redox Biology, vol. 19, pp. 339-353, 2018.

[80] S. Chen, X. Lv, B. Hu et al., "Critical contribution of RIPK1 mediated mitochondrial dysfunction and oxidative stress to compression-induced rat nucleus pulposus cells necroptosis and apoptosis," Apoptosis, vol. 23, no. 5-6, pp. 299-313, 2018.

[81] J. Montane, A. Klimek-Abercrombie, K. J. Potter, C. Westwell-Roper, and C. Bruce Verchere, "Metabolic stress, IAPP and islet amyloid," Diabetes, Obesity and Metabolism, vol. 14, pp. 68-77, 2012.

[82] X. Wu, K. Wang, W. Hua et al., "Down-regulation of islet amyloid polypeptide expression induces death of human annulus fibrosus cells via mitochondrial and death receptor pathways," Biochimica et Biophysica Acta (BBA) - Molecular Basis of Disease, vol. 1863, no. 6, pp. 1479-1491, 2017.

[83] X. Li, F. R. Wu, R. S. Xu et al., “Acid-sensing ion channel 1Amediated calcium influx regulates apoptosis of endplate chondrocytes in intervertebral discs," Expert Opinion on Therapeutic Targets, vol. 18, no. 1, pp. 1-14, 2014.

[84] P. J. Roughley, "Biology of intervertebral disc aging and Degeneration," Spine, vol. 29, no. 23, pp. 2691-2699, 2004.

[85] S. Gonzales, C. Wang, H. Levene, H. S. Cheung, and C.-Y. C. Huang, "ATP promotes extracellular matrix biosynthesis of intervertebral disc cells," Cell and Tissue Research, vol. 359, no. 2, pp. 635-642, 2015.

[86] X. Yin, S. Gonzales, S. Sha, H. Levene, and C. Y. Huang, "The effect of adenosine on extracellular matrix production in porcine intervertebral disc cells," Cells, Tissues, Organs, vol. 206, no. 1-2, pp. 73-81, 2019.

[87] L. Yang, Z. Rong, M. Zeng et al., "Pyrroloquinoline quinone protects nucleus pulposus cells from hydrogen peroxideinduced apoptosis by inhibiting the mitochondria-mediated pathway," European Spine Journal, vol. 24, no. 8, pp. 1702$1710,2015$.

[88] Y. Chen, Y. Wu, H. Shi et al., "Melatonin ameliorates intervertebral disc degeneration via the potential mechanisms of mitophagy induction and apoptosis inhibition," Journal of Cellular and Molecular Medicine, vol. 23, no. 3, pp. 21362148, 2019. 
[89] H. Lin, X. Ma, B.-C. Wang et al., "Edaravone ameliorates compression-induced damage in rat nucleus pulposus cells," Life Sciences, vol. 189, pp. 76-83, 2017.

[90] N. Fujita, S. S. Gogate, K. Chiba, Y. Toyama, I. M. Shapiro, and M. V. Risbud, "Prolyl hydroxylase 3 (PHD3) modulates catabolic effects of tumor necrosis factor- $A$ (TNF- $\alpha$ ) on cells of the nucleus pulposus through co-activation of nuclear factor $\kappa \mathrm{B}(\mathrm{NF}-\kappa \mathrm{B}) / \mathrm{p} 65$ signaling," The Journal of Biological Chemistry, vol. 287, no. 47, pp. 39942-39953, 2012.

[91] A. Hiyama, K. Yokoyama, T. Nukaga, D. Sakai, and J. Mochida, "A complex interaction between Wnt signaling and TNF- $\alpha$ in nucleus pulposus cells," Arthritis Research \& Therapy, vol. 15, no. 6, p. R189, 2013.

[92] Y. Tian, W. Yuan, N. Fujita et al., "Inflammatory cytokines associated with degenerative disc disease control aggrecanase-1 (ADAMTS-4) expression in nucleus pulposus cells through MAPK and NF- $\kappa \mathrm{B}$," The American Journal of Pathology, vol. 182, no. 6, pp. 2310-2321, 2013.

[93] R. M. Mason and C. Sweeney, "The relationship between proteoglycan synthesis in swarm chondrocytes and pathways of cellular energy and UDP-sugar metabolism," Carbohydrate Research, vol. 255, pp. 255-270, 1994.

[94] B. P. Dranka, B. G. Hill, and V. M. Darley-Usmar, "Mitochondrial reserve capacity in endothelial cells: the impact of nitric oxide and reactive oxygen species," Free Radical Biology and Medicine, vol. 48, no. 7, pp. 905-914, 2010.

[95] P. H. Reddy, X. L. Yin, M. Manczak et al., "Mutant APP and amyloid beta-induced defective autophagy, mitophagy, mitochondrial structural and functional changes and synaptic damage in hippocampal neurons from Alzheimer's disease," Human Molecular Genetics, vol. 27, no. 14, pp. 2502-2516, 2018.

[96] X. Yao, J. Zhang, X. Jing et al., "Fibroblast growth factor 18 exerts anti-osteoarthritic effects through PI3K-AKT signaling and mitochondrial fusion and fission," Pharmacological Research, vol. 139, pp. 314-324, 2019.

[97] M. Y. Ansari, N. M. Khan, I. Ahmad, and T. M. Haqqi, "Parkin clearance of dysfunctional mitochondria regulates ROS levels and increases survival of human chondrocytes," Osteoarthritis and Cartilage, vol. 26, no. 8, pp. 1087-1097, 2018.

[98] H. Lin, L. Zhao, X. Ma et al., "Drp 1 mediates compressioninduced programmed necrosis of rat nucleus pulposus cells by promoting mitochondrial translocation of P 53 and nuclear translocation of AIF," Biochemical and Biophysical Research Communications, vol. 487, no. 1, pp. 181-188, 2017.

[99] Y. Chen, J. Lin, J. Chen et al., "Mfn 2 is involved in intervertebral disc degeneration through autophagy modulation," Osteoarthritis and Cartilage, vol. 28, no. 3, pp. 363-374, 2020.

[100] Y. Wang, J. Shen, Y. Chen et al., "PINK1 protects against oxidative stress induced senescence of human nucleus pulposus cells via regulating mitophagy," Biochemical and Biophysical Research Communications, vol. 504, no. 2, pp. 406-414, 2018.

[101] J. Lin, J. Zhuge, X. Zheng et al., "Urolithin A-induced mitophagy suppresses apoptosis and attenuates intervertebral disc degeneration via the AMPK signaling pathway," Free Radical Biology and Medicine, vol. 150, pp. 109-119, 2020.

[102] W.-N. Xu, H.-L. Zheng, R.-Z. Yang et al., "Mitochondrial NDUFA4L2 attenuates the apoptosis of nucleus pulposus cells induced by oxidative stress via the inhibition of mitophagy," Experimental \& Molecular Medicine, vol. 51, no. 11, pp. 1-16, 2019.
[103] R. Schreck, P. Rieber, and P. A. Baeuerle, "Reactive oxygen intermediates as apparently widely used messengers in the activation of the NF-kappa B transcription factor and HIV1,” The EMBO Journal, vol. 10, no. 8, pp. 2247-2258, 1991.

[104] K. G. Ma, Z. W. Shao, S. H. Yang et al., "Autophagy is activated in compression-induced cell degeneration and is mediated by reactive oxygen species in nucleus pulposus cells exposed to compression," Osteoarthritis and Cartilage, vol. 21, no. 12, pp. 2030-2038, 2013.

[105] S. Suzuki, N. Fujita, N. Hosogane et al., "Excessive reactive oxygen species are therapeutic targets for intervertebral disc degeneration," Arthritis Research \& Therapy, vol. 17, no. 1, p. 316, 2015 .

[106] C. R. Powell, K. M. Dillon, and J. B. Matson, "A review of hydrogen sulfide $\left(\mathrm{H}_{2} \mathrm{~S}\right)$ donors: Chemistry and potential therapeutic applications," Biochemical Pharmacology, vol. 149, pp. 110-123, 2018.

[107] R. Wedmann, S. Bertlein, I. Macinkovic et al., "Working with " $\mathrm{H}_{2} \mathrm{~S}$ ": Facts and apparent artifacts," Nitric Oxide, vol. 41, pp. 85-96, 2014.

[108] B. Murphy, R. Bhattacharya, and P. Mukherjee, "Hydrogen sulfide signaling in mitochondria and disease," FASEB Journal, vol. 33, no. 12, pp. 13098-13125, 2019.

[109] C.-x. Qi, Q. Zhou, Z. Yuan et al., "Kinsenoside: a promising bioactive compound from Anoectochilus species," Current Medical Science, vol. 38, no. 1, pp. 11-18, 2018.

[110] Y. Wang, R. Zuo, Z. Wang et al., "Kinsenoside ameliorates intervertebral disc degeneration through the activation of AKT-ERK1/2-Nrf2 signaling pathway," Aging, vol. 11, no. 18, pp. 7961-7977, 2019.

[111] B. Zhang, L. Xu, N. Zhuo, and J. Shen, "Resveratrol protects against mitochondrial dysfunction through autophagy activation in human nucleus pulposus cells," Biochemical and Biophysical Research Communications, vol. 493, no. 1, pp. 373-381, 2017.

[112] X. Li, F. M. Phillips, H. S. An et al., "The action of resveratrol, a phytoestrogen found in grapes, on the intervertebral disc," Spine, vol. 33, no. 24, pp. 2586-2595, 2008.

[113] W. Hua, S. Li, R. Luo et al., "Icariin protects human nucleus pulposus cells from hydrogen peroxide-induced mitochondriamediated apoptosis by activating nuclear factor erythroid 2related factor 2," Biochimica et Biophysica Acta (BBA) - Molecular Basis of Disease, vol. 1866, no. 1, p. 165575, 2020.

[114] G. F. Kelso, C. M. Porteous, C. V. Coulter et al., "Selective targeting of a redox-active ubiquinone to mitochondria within cells: antioxidant and antiapoptotic properties," Journal of Biological Chemistry, vol. 276, no. 7, pp. 4588-4596, 2001.

[115] S. Rodriguez-Cuenca, H. M. Cochemé, A. Logan et al., "Consequences of long-term oral administration of the mitochondria-targeted antioxidant MitoQ to wild-type mice," Free Radical Biology and Medicine, vol. 48, no. 1, pp. 161-172, 2010.

[116] A. Dhanasekaran, S. Kotamraju, S. V. Kalivendi et al., "Supplementation of endothelial cells with mitochondriatargeted antioxidants inhibit peroxide-induced mitochondrial iron uptake, oxidative damage, and apoptosis," Journal of Biological Chemistry, vol. 279, no. 36, pp. 37575-37587, 2004.

[117] R. A. J. Smith, C. M. Porteous, A. M. Gane, and M. P. Murphy, "Delivery of bioactive molecules to mitochondria in vivo," Proceedings of the National Academy of Sciences of 
the United States of America, vol. 100, no. 9, pp. 5407-5412, 2003.

[118] E. F. Fang, Y. Hou, K. Palikaras et al., "Mitophagy inhibits amyloid- $\beta$ and tau pathology and reverses cognitive deficits in models of Alzheimer's disease," Nature Neuroscience, vol. 22, no. 3, pp. 401-412, 2019.

[119] X. Onphachanh, H. J. Lee, J. R. Lim et al., "Enhancement of high glucose-induced PINK1 expression by melatonin stimulates neuronal cell survival: involvement of MT2/Akt/NF- $\kappa \mathrm{B}$ pathway," Journal of Pineal Research, vol. 63, no. 2, article e12427, 2017.

[120] W.-N. Xu, R.-Z. Yang, H.-L. Zheng et al., "PGC-1 $\alpha$ acts as an mediator of Sirtuin2 to protect annulus fibrosus from apoptosis induced by oxidative stress through restraining mitophagy," International Journal of Biological Macromolecules, vol. 136, pp. 1007-1017, 2019.

[121] C. Ricca, A. Aillon, L. Bergandi, D. Alotto, C. Castagnoli, and F. Silvagno, "Vitamin D receptor is necessary for mitochondrial function and cell health," International Journal of Molecular Sciences, vol. 19, no. 6, p. 1672, 2018.

[122] R. A. H. van de Ven, D. Santos, and M. C. Haigis, "Mitochondrial sirtuins and molecular mechanisms of aging," Trends in Molecular Medicine, vol. 23, no. 4, pp. 320-331, 2017.

[123] A. T. Dinkova-Kostova and A. Y. Abramov, "The emerging role of Nrf2 in mitochondrial function," Free Radical Biology and Medicine, vol. 88, Part B, pp. 179-188, 2015.

[124] Z. Tang, B. Hu, F. Zang, J. Wang, X. Zhang, and H. Chen, "Nrf2 drives oxidative stress-induced autophagy in nucleus pulposus cells via a Keap1/Nrf2/p62 feedback loop to protect intervertebral disc from degeneration," Cell Death \& Disease, vol. 10, no. 7, p. 510, 2019. 